\title{
INVESTIGANDO O ENSINO DA CULTURA NA SALA DE AULA DE LÍNGUA ESTRANGEIRA
}

\section{Glorinha Guerreiro (*) Douglas Altamiro ${ }^{(*)}$}

Resumo: O objetivo deste trabalho é verificar o aspecto do ensino da cultura em sala de aula de LE, como este ensino é feito de modo a auxiliar os aprendizes no enfrentamento de problemas multiculturais que podem surgir dentro de uma realidade globalizada. A pesquisa, de cunho etnográfico, teve como participante uma professora formada em uma universidade brasileira, nunca tendo estado no exterior e, portanto, tendo adquirido todo seu conhecimento da língua-alvo no Brasil. Notas de campo, gravações em áudio e vídeo e entrevistas foram os instrumentos utilizados para analisar sua competência lingüística na língua-alvo, considerando a perspectiva cultural dentro de sua prática.

Palavras-chave: cultura; linguagem; ensino de língua estrangeira

\section{Introdução}

Este trabalho procura investigar a cultura relacionada ao ensino da língua estrangeira (doravante LE) dentro de contextos de comunicação, isto é, enquanto a cultura é veiculada no uso da LE.

O ensino da LE pode ser, para o professor, o ensino das estruturas da língua (ou o ensino das quatro habilidades de uma forma mais estruturada), e pode ser também o ensino da LE enquanto veiculadora de uma outra cultura (de uma outra visão de mundo).

A LE enquanto veiculadora de uma outra cultura é uma questão importante, pois, atualmente, com a globalização, os

Glorinha Mendonça da Silva Guerreiro e Douglas Altamiro Consolo Universidade Estadual Paulista - Unesp, São José do Rio Preto - SP

Comunicação apresentada em 2004 no $14^{\circ}$ Inpla - Intercâmbio de Pesquisas em Lingüística Aplicada, Simpósio Cultura e Ensino de Línguas, PUC/ SP. 
indivíduos podem ter acesso a meios que os colocam expostos às questões multiculturais, como por exemplo, no trabalho, na escola, pelos meios de comunicação de massa, pela Internet. Deste modo, os alunos podem vir para a sala de aula trazendo dúvidas relativas a como lidar com diferenças culturais, tanto no nível de apropriação no uso das palavras, quanto no nível de diferentes visões de mundo encontradas nos diversos povos.

Dois pontos que podem ser considerados no que se refere à consciência cultural é que, primeiro, a falta de informação a respeito de certos aspectos culturais pode acarretar choques culturais no confronto com o contexto onde ela é utilizada.

Segundo, por meio do encontro com língua e culturas estrangeiras, pode ser suscitada uma compreensão da cultura do próprio indivíduo, no que se refere ao modo de utilizar as palavras na língua nativa e na visão que o povo tem no modo de relacionar palavras e situações, por exemplo.

Organização do artigo

Este artigo será apresentado, primeiramente, com a exposição da metodologia de pesquisa, procurando descrever a sua natureza, o contexto, os participantes, os instrumentos utilizados para a coleta de dados e os procedimentos de análise. Depois será apresentado o arcabouço teórico, feito por meio da apresentação das teorias que norteiam este trabalho. A seguir, será feita a análise e discussão dos dados, encaminhando-se para a conclusão.

Objetivo

O objetivo deste trabalho é investigar como um professor de LE proporciona aos alunos o contato com uma cultura diferente, no que se refere ao ensino da língua; como este contato é trabalhado em sala de aula e como o professor pode suscitar situações de reflexão sobre a cultura da LE, comparando-a, possivelmente, com a cultura da língua nativa dos alunos.

Para atingir este objetivo, foi verificada a prática do professor em sala de aula, o tratamento do conteúdo e a utilização do material didático, bem como o uso da linguagem. 
Foi também verificado, como, na visão do professor, a formação pré-serviço contribuiu para a sua prática no que se refere aos aspectos sócio-culturais.

\section{Participante da pesquisa e contexto investigado}

O participante da pesquisa é uma professora formada em uma universidade pública do interior do Estado de São Paulo, na área de Licenciatura em Letras.

Um detalhe importante para esta pesquisa é que a professora nunca esteve fora do Brasil. Este fato contribui para que se possa verificar quais são as fontes de informação que ela utiliza para abordar os aspectos sócio-culturais em sala de aula.

O contexto investigado foi o de uma escola que oferece cursos livres de idiomas e os alunos eram de nível intermediário.

Metodologia de pesquisa

Foi desenvolvida uma pesquisa interpretativista, isto é, uma pesquisa de cunho etnográfico. A pesquisa etnográfica, segundo Watson-Gegeo (1988, p.38), é o estudo do comportamento das pessoas em ambiente natural, com foco na interpretação cultural do comportamento, sendo que o seu objetivo é a descrição e interpretação do que as pessoas fazem nesse ambiente, os resultados de suas interações e a visão que elas têm de suas atividades.

No que se refere à metodologia, ainda segundo Watson-Gegeo (id. ibid. p, 43), a pesquisa etnográfica inclui técnicas de observação, entrevistas, gravações de áudio e vídeo.

Instrumentos de pesquisa

Inicialmente foi aplicado um questionário com perguntas abertas à professora participante com a finalidade de verificar as suas crenças quanto ao ensino da cultura, suas afinidades com a cultura da língua-alvo, a importância dada a esse item em sua prática e como foi o aprendizado da cultura da língua-alvo em seu período de formação pré-serviço, além de verificar como é o seu contato com a LE no contexto brasileiro. 
A seguir, iniciou-se a pesquisa de campo com observações sistemáticas das aulas e notas de campo, acompanhadas de diários de observação, elaborados pelo pesquisador ao final de cada aula assistida, com a finalidade de fixar melhor o contexto e a situação observada e poder iniciar uma análise, buscando pistas de como essas observações poderiam responder às perguntas de pesquisa.

Gravações em áudio e em vídeo foram também utilizadas com c objetivo de obter registros mais detalhados sobre os fenômenos ocorridos no contexto de sala de aula.

Uma análise preliminar do questionário suscitou perguntas que serviram para aprofundamento de questões vistas como cruciais para a investigação. Foi feita, então, uma entrevista com a professora, com gravação em áudio. Essa entrevista foi acompanhada de uma sessão de visionamento, onde foi possível confrontar algumas crenças da professora e sua prática.

Finalmente, foi efetuada a triangulação dos dados. Segundo Watson-Gegeo (1988, p. 45) a triangulação dos dados, isto é, o juntar as informações advindas de diferentes fontes, é uma estratégia importante para se chegar à validação das descobertas feitas no trabalho etnográfico.

Perguntas de pesquisa

Esta investigação está sendo orientada pelas seguintes perguntas de pesquisa:

1. Como se apresenta o ensino de aspectos culturais de um professor de LE em sala de aula?

2. Como o professor relaciona o conteúdo de sua formação pré-serviço com sua prática de sala de aula voltada aos aspectos culturais?

Arcabouço teórico

O ensino de uma LE tem sido visto, por alguns autores (por exemplo, Consolo, 2002; Hinkel, 1999; Kramsch, 1994; Brown, 1987), como inseparável do ensino de sua cultura. Estudos têm sido feitos para encontrar o lugar da cultura no aprendizado de LE e tem se verificado sua importância no contexto de sala de aula. 
Brown (1987) ressalta a importância da cultura na vida dos seres humanos afirmando que a "cultura é um modo de vida", ela é "o contexto no qual nós existimos, pensamos, sentimos e nos relacionamos com os outros". Para o autor, cultura é uma espécie de “"cola' que mantém um grupo de pessoas juntas".

Brown (id., p. 122.) cita a definição que Larson \& Smalley (1972) dão de cultura. Segundo esses autores, cultura seria a 'maquete' que

guia o comportamento das pessoas em uma comunidade e está incubada na vida familiar. Ela governa nosso comportamento em grupos, nos faz sensíveis às questões de status e nos ajuda a saber o que os outros esperam de nós e o que acontecerá se nós não correspondermos às suas expectativas. A cultura nos ajuda a saber até onde podemos ir como indivíduos e qual é a nossa responsabilidade no grupo. Diferentes culturas são as bases estruturais que fazem uma comunidade redonda ser redonda e uma comunicade quadrada ser quadrada.'

Para Brown (ibid.), a cultura não é apenas um conjunto de idéias, costumes, artes e ferramentas que caracterizam um grupo de pessoas mas é também um componente que preenche algumas necessidades psicológicas e biológicas dos seres humanos.

O autor coloca também uma definição de percepção como sendo algo que envolve o ato de "filtrar a informação mesmo antes de ela ser guardada na memória”. Então, o que pareceria a alguém como uma percepção interessante, por exemplo, sobre uma outra pessoa ou

Minha tradução para o trecho citado:

'...'blueprint' that guides the behavior of people in a community and is incubated in family life. It governs our behavior in groups, makes us sensitive to matters of status, and helps us know what others expet of us and what will happen if we do not live up to their expectations. Culture helps us to know how far we can go as individuals and what our responsability is to the group. Different cultures are the underlying structures which make Round community round and Square community square." 
uma idéia, é, algumas vezes, desinteressante para alguém de uma outra cultura e daí podem surgir males-entendidos.

Por todos os fatores apontados acima, Brown (id.) afirma não conceber o ensino de uma LE sem o ensino de sua cultura. Para ele, "uma língua é parte de uma cultura e uma cultura é parte de uma língua; as duas estão tão intrinsicamente ligadas que não se pode separar as duas sem perder o significado tanto de uma quanto da outra". (p.123) ${ }^{2}$

A relação entre cultura e ensino de LE é bastante intrínseca. Segundo Kramsch (1998, p. 3), a língua utilizada em contextos de comunicação é conectada à cultura de várias formas. Uma dessas formas é a linguagem expressando uma realidade cultural, isto é, pessoas transmitindo idéias, faîos ou eventos que são comunicáveis no sentido de que expressam um conhecimento de mundo compartilhado por um mesmo grupo.

Uma outra forma seria o modo como as pessoas se expressam no meio visual, falado ou escrito, criando significados que são compreensíveis para pessoas de um mesmo grupo. Por esses aspectos verbais e não-verbais a linguagem estaria incorporando uma realidade cultural. Uma última forma seria a linguagem simbolizando uma realidade cultural. As pessoas identificam-se e identificam os outros através da linguagem e, neste sentido, a linguagem seria um símbolo de identidade social.

Essa relação intrínseca entre cultura e linguagem traz para o professor a tarefa de conduzir seus alunos, por meio do aprendizado de uma LE, ao contato não só com as formas lingüísticas mas também ao contato com uma realidade cultural diversa.

2 Minha tradução para o trecho:

"A language is part of a culture and a culture is part of a language; the two are intricately interwoven such that one cannot separate the two without losing the significance of either language or cuiture". 
Kramsch (1998, p.10) define cultura como sendo os padrões de percepções, crenças, valores e atos que uma pessoa carrega consigo. Essa pessoa, enquanto membro de uma comunidade discursiva que tem em comum o espaço social, a história e as imagens, retém consigo os padrões culturais de sua comunidade mesmo quando ela deixa de conviver nessa comunidade.

A autora (id ibid.) aponta alguns aspectos da cultura. O primeiro aspecto é o fato de que a cultura é algo acrescentado à natureza humana, ela não nasce com o homem, isto é, é o resultado de uma intervenção humana nos processos biológicos da natureza.

Um segundo aspecto é o fato de que a cultura, ao mesmo tempo em que liberta, ela impõe algo à vida do homem. Ela liberta porque investe a vida do homem de uma identidade. Por exemplo, a família ou o meio profissional formam a identidade do homem no sentido de que fornece um modo comum de ver o mundo; nesse sentido, as pessoas têm atitudes, crenças e valores comuns.

Ao mesmo tempo, a cultura impõe os traços comuns que devem ser utilizados para que uma pessoa seja identificada com um certo grupo. Por exemplo, os adolescentes têm suas gírias ou o meio profissional tem os seus jargões e quem não pertence a esses grupos não consegue muitas vezes compreendê-los. Esses dois princípios, o da liberdade e o da imposição, afetam o homem no seu plano social, histórico e metafórico, isto é, das imagens das coisas que o homem cria.

A cultura também estaria situada no discurso comunitário, imaginado pelo homem, criado e moldado pela linguagem.

O patrimônio cultural serviria para perpetuar relações de poder e de dominação, que distinguiria classes ou grupos sociais e culturas dominantes. A autora dá exemplos de alguns estudos que mostram os efeitos hegemônicos das culturas dominantes e da autoridade que representam para o 'outro'. Para ela, levar uma cultura a sério significa questionar a base intelectual de um grupo e aceitar o fato de que o conhecimento é preenchido do contexto social e histórico no qual ele é adquirido e disseminado. A esse respeito, o estudo de uma língua é uma atividade eminentemente cultural. 
Um último aspecto da cultura apontado por Kramsch (1998) é o da heterogeneidade, isto é, membros de uma mesma cultura possuem diferenças de idade, sexo, étnicas, políticas, de experiências de vida. Além disso, a cultura é algo que está em constante mudança e por esses dois fatores, as culturas estão sempre em constante luta por reconhecimento e legitimação.

Eli Hinkel, em seu livro Cuiture in Second Language Teaching and Learning (1999), faz, na Introdução, um apanhado dos trabalhos que têm sicio feitos até o momento sobre a questão da cultura e do aprendizado de LE. Ele cita Rosaldo (1984), que afirma que "cultura é mais do que um mero catálogo de rituais e crenças". Para a autora, a cultura deriva do mundo, no qual as pessoas vivem, e da realidade que ela constróem . (p. 1)

Hinkel (ibid.) cita também Thomas $(1983,1984)$, que observa que "falantes não nativos são pegos freqüentemente adotando comportamento lingüísticos não apropriados e muitas vezes eles não têm consciência do que está acontecendo, para a autora, esse tipo de violação de normas culturais leva a falhas sociopragmáticas, quebras de comunicação e estereotipização de falantes não nativos". (p. 2)

Como é possível observar nas definições acima, cultura não é apenas um conjunto de rituais, costumes, artes, gestos, enfim tudo o que parece estar apenas na manifestação visual, ela é também algo que está ligado à constituição do ser humano. Não se pode pensar no ser humano sem também pensar na sua cultura. E o desconhecimento da cultura do 'outro' pode levar a problemas de falhas sociopragmáticas, quebra de comunicação e esteriotipização citados por Thomas (op. cit.).

Essa intrínseca ligação entre cultura e ensino de LE remete ao encontro de duas ou mais culturas, gerando o espaço da transculturalidade.

Kramsch (1998, p. 81) explica que o termo transcultural refere-se a culturas diferentes que se encontram fora dos limites de cada nação e nesse encontro pode haver um choque cultural. Para que seja evitado esse choque, o ensino de língua estrangeira com abordagem transcultural procura a compreensão do 'outro' por meio do aprendizado de sua língua. Essa junção da própria cultura com a 
cultura do 'outro' promove uma nova visão dessa cultura da línguaalvo vinda dos aprendizes. Segundo Kramsch (mimeo),

Usuários de outras línguas além de sua própria língua podem revelar caminhos inesperados ao lidar com problemas transculturais que eles encontram quando migram entre as línguas. A sua apropriação de línguas estrangeiras os permite construir identidades linguírsticas e culturais nas intersecções das línguas nacionais e às margens dos territórios dos falantes monolíngües. ${ }^{3}$

A autora (ibid.) acrescenta ainda, que:

Vista da perspectiva da viagem lingüística e da migração mais do que daquela do sedentarismo tradicional, oposição limitada entre nativo e não nativo, a noção de falante nativo perde sua força e significação. Muito mais interessantes são as múltiplas possibilidades de expressão própria na língua. ${ }^{4}$

Análise dos dados

a) Categorias

As categorias surgiram a partir dos objetivos desta pesquisa, que são a investigação de:

1. Prática do professor em sala de aula

2. Tratamento do conteúdo

3 Minha tradução para o trecho:

"Users of tongues other than their own can reveal unexpected ways of dealing with the cross-cultural clashes they encounter as they migrate between languages. Their appropriation of foreign languages enables them to construct linguistic and cultural identities in the interstices of national languages and on the margins of monolingual speakers' territories."

4 Minha tradução para o trecho:

"Seen from the perspective of linguistic travel and migration rather than from that of the traditional sedentary, bounded opposition native/nonnative, the notion of native speakership loses its power and significance. Far more interesting are the multiple possibilities for selfexpression in language." 
3. Utilização do material didático

4. Uso da linguagem

5. Formação pré-serviço

A partir destas categorias surgiram algumas micro-categorias, que serão analisadas neste trabalho.

A investigação das quatro primeiro categorias auxiliarão na tentativa de responder a primeira pergunta de pesquisa.

b) Micro-categorias

Dentro da primeira categoria - prática do professor em sala de aula -, foi encontrada uma micro-categoria, que consiste numa tentativa de contraposição entre a cultura da LE e cultura da língua materna em sala de aula.

Ao responder ao questionário, a professora (doravante $\mathrm{P} 1$ ) colocou-se a favor da apresentação da cultura da língua estrangeira por meio de uma contraposição entre a cultura da língua materna e a cultura da LE.

O segmento abaixo confirma essa posição:

Segmento 1

"Sim. $O$ aluno que entra em contato com uma $L E$, a meu ver, tem a tendência de supervalorizar a cultura estrangeira em detrimento da sua própria cultura (música, modo de se vestir, etc., principalmente). Por isso, o professor deve sempre contrapor a cultura da LM com a de LE." (Quest-P1)

Em uma das aulas observadas, P1 procurou trabalhar, estabelecendo uma comparação entre a cultura da LE e a cultura da língua materna. Porém, este trabalho foi baseado nas opiniões dos alunos a respeito do conteúdo proposto. Por meio da observação da aula, percebe-se que P1 não tem informações mais sólidas a respeito do sentido da palavra "favelas" na língua inglesa.

Os segmentos abaixo ilustram as afirmações acima:

Segmento 2: Comparação entre aspectos culturais relacionados ao Brasil e aos países de língua inglesa 


\section{DE LINNGUA ESTRANGEIRA}

$P$ pede informação e opinião dos alunos, um aluno responde:

$\mathrm{P}: \quad / \ldots /$ for example $(+)$ do they have favelas in Britain (+) in Britain",

A3: they have

$\mathrm{P}: \quad$ hum hum $(+)$ favelas

A: favelas

P: I used this word (+) favelas (+) because they use ( it ) in Simpsons (+) do they have favelas in Britain $(+)$ or in the United States",

A4: I don't know

A2: $\quad$ not like us $(+)$ but they ã: have a kind

Alunos dão a informação, expressam suas opiniões e discutem a questão sobre favelas nos paises de língua inglesa:

A 1: gueto

A2: maybe in the (INCOMP) have a division about white and black people and the black have a division like us (+) eh: like to our favela

Segmento 2 - Parte 2:

Alunos fornecem informação, expressam opinião e discutem o assunto favelas nos paises de língua inglesa:

/...

A3: I don't know because when you saw $(+)$ watch $(+)$ watch a film in New York erm (+) always see some people under the bridges in the $\tilde{a}:(+)$ in the streets ã: $(+)$ they ã: $(+)$ like Brazil

A2: but how many people have you seen in a film that lives on the streets?

A3: I think

A2: how many in Brazilian films?

A3: I think if you travel to United States or New York you (+) go to see people in the streets (INCOMP)

Ao questionar o sentido da palavra "favelas" na língua inglesa, um professor de origem inglesa, que ministra aulas na universidade, explicou-me que a palavra "favelas" é utilizada na língua inglesa, escrita do mesmo modo como escreve-se na língua portuguesa, especificamente para textos que se referem ao Brasil. 
Na Inglaterra não existem "favelas", existem "homeless people", pessoas que moram nas ruas.

$\mathrm{Na}$ língua inglesa existe também o termo "chanty town", que seria um conjunto de residências construídas com materiais descartáveis, também não existentes na Inglaterra. Um exemplo de "chanty town" seria "Trench Town" (Jamaica).

"Slums" é uma outra palavra utilizada para expressar cortiços, isto é, prédios abandonados que são invadidos. Úm exemplo de "slums" são os "Gorbals" (Glasgow).

Este tipo de informação poderia completar a lacuna entre o que os alunos acham e o que é a realidade, oferecendo a oportunidade para uma contraposição entre as duas culturas, em que os alunos pudessem olhar a sua realidade e a realidade da língua estrangeira, verificando as diferenças e as similaridades com mais informações a respeito do assunto.

A segunda categoria - tratamento do conteúdo, revelou uma micro-categoria a respeito de estereótipos. Uma das aulas observadas tem como conteúdo afirmativas do aluno a respeito da visão do americano com relação ao Brasil, que encontrou respaldo na posição de P1 em sala de aula.

Durante a entrevista, P1 afirmou que a criação de estereótipos vem da visão errada que cada povo tem do outro, ela afirma que é difícil quebrar estes estereótipos e, em sessão de visionamento, ao assistir a um trecho gravado da aula, onde o aluno fala sobre a "visão americana de ver o Brasil", ela afirma que concorda com a posição do aluno, mas que deveria ter trazido outras formas de fazer a comparação entre as culturas.

Os segmentos abaixo mostram essas questões:

Segmento 3

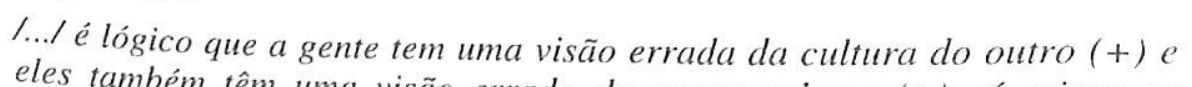
eles também têm uma visão errada da nossa cultura $(+)$ aí criam os estereótipos e é é difícil tirar esses estereótipos dos alunos e até mesmo
deles sobre a gente ou essa é uma visão nossa sobre eles $(+)$ é difícil quebrar esses estereótipos na aula $(+)$ por isso eu acho que: (+) trabalhar sempre com eh: (+) uma comparação das duas culturas aí ajuda $(+)$ mostrar que não é a nossa visão $(+)$ da cultura deles por 


\section{DE LINGUA ESTRANGEIRA}

exemplo (+) pegar estereótipos de outros outros países sobre eles (+) aí sim ajuda desde que seja feita de forma comparativa /.../(Entr-P1)

Segmento 4: Estereótipos

P pergunta sobre o assunto e pede informações aos alunos:

P: $\quad / \ldots /$ have you seen Simpsons $(+)$ that episode that tells about Brazil"' $(+)$ what's $(+)$ what's the image they $(+)$ show about our country",

Um aluno responde, $P$ e os alunos discutem o assunto estereótipos sobre o Brasil:

A2: the image is Brazil is a jungle

P : everywhere',

A2: everywhere

P : or just Amazon", (+) or

A: everywhere

A2: $\quad$ is the American way of see us

A3: monkeys

$\mathrm{P}: \quad$ in the middle of the street

A3: (INCOMP)

A2: and the Brazilian capital is Buenos Aires

\section{Segmento 5}

/.../ do que jeito que teve lá nessa aula em específico (+) eu acho que só contribui pra: (+) aumentar o estereótipo (+) né" " $(+)$ eu vi que não eu não desmistifiquei aquilo essa visão americana $(+)$ eu concordo é mesmo $(+)$ mas eu não trouxe outras visões $(+)$ né," $(+)$ sobre a nossa cultura (visionamento-PI)

Na terceira categoria - utilização do material didático -, foi encontrada uma micro-categoria referente à apresentação da cuítura estrangeira baseada no livro didático.

Um exemplo nesta micro-categoria foi uma proposta de atividade a respeito dos funerais na China, que foi trazida pelo livro didático.

Nesta aula, P1 estabeleceu a comparação entre a cultura chinesa e a cultura brasileira a respeito de funerais, obtendo êxito na 
sua proposta ao questionar os alunos sobre a cultura brasileira e propôr a leitura, a observação das figuras e a atividade de compreensão oral trazidas no livro a respeito da China.

\section{Segmento 6}

$P$ pergunta sobre o assunto e pede informações aos alunos:

P: $\quad$ so in Brazil what we do when a person die" (+) during the funeral'"

A4: when somebody die",

P : ya

$$
\text { I.../ }
$$

A3: we call a padre

A : priest

$$
\text { l.../ }
$$

A : $\quad$ we get sad

P : $\quad$ we get sad yes (+) for sure what else'" (+) finish",

A: $\quad$ ã: we: we wore $(+)$ dark clothes

Segmento 6 - Parte 2

P: what about China do you know" what they do there', (++) take a look at these pictures (++) what are they doing",

A4: they are praying or something like that

P : praying ok" they are (+) maybe singing ok" They play flute l...l

P: what are what is the colour of the clothes?

A: $\quad$ white $(++)$ snow white

$\mathrm{Na}$ quarta categoria - uso da linguagem, foram observadas algumas dificuldades que a professora tem quanto ao esclarecimento de dúvidas a respeito de vocabulário.

Apenas para efeito de ilustração, apresento, no segmento abaixo, uma das dificuldades encontradas e registrada no diário de observação, referente à palavra "atestado de óbito": 


\section{DE LÍNGUA ESTRANGEIRA}

\section{Segmento 7}

Durante a leitura dos textos, eles discutiram sobre o assunto criogenia e deram suas opiniões. Uma outra palavra que surgiu durante essa discussão foi "atestado de óbito" que A2 tentou relacionar com "obit paper". Pl não sabia a expressão em inglês e não pôde ajudá-lo. (Diário-11/06)

Durante a entrevista, ao ser questionada sobre o assunto, a professora coloca a dificuldade a respeito de pesquisar questões de vocabulário. O segmento abaixo coloca a questão.

\section{Segmento 8}

Pl: bom (+) é: é complicado porque a gente não sabe tudo $(+) n e ́ ")(+) e$ sempre surge l...l ai eu acho que indo lá e pesquisando e trazendo em aulas $(+)$ posteriores acho que seria o ideal (+) nem sempre consigo porque às vezes (+) uma dívida se você não anota ali (+) né" (+) acaba se perdendo (+) e: (+) ao mesmo tempo se você pára (+) anota (+) né" (+) não dá prosseguimento àquela aula específica (+) então fica o dilema /.../ (Entr-Pl)

Para responder a segunda pergunta de pesquisa, sobre a formação pré-serviço da professora, procurei apoiar-me nas declarações de P1 feitas por meio do questionário e da entrevista, onde pude detectar suas opiniões a respeito de como foi o seu aprendizado no que se refere aos aspectos culturais.

No questionário, ao ser perguntada a respeito da importância do ensino de aspectos culturais em um curso de formação de professores, P1 respondeu de forma afirmativa. Para ela, o ensino de aspectos culturais durante a graduação permite a comparação da cultura da LE com a cultura da LM. Isto traria como consequiência uma maior conscientização dos alunos com relação a ambas as culturas.

Ainda em resposta ao questionário, durante o período em que estava na graduação, P1 afirmou que o ensino de aspectos culturais foi contemplado, porém, não de forma muito explícita. O segmento abaixo é referente a esta questão: 


\section{Segmento 9}

l.../ houve um texto sobre o comportamento não verbal dos falantes de inglês; aspectos religiosos, culinários e culturais também foram abordados, mas faltou muita coisa. Sempre falta e depende do aluno buscar isso. (Quest-P1)

Durante a entrevista, P1 afirmou, ainda que, como não há um curso específico de cultura inglesa durante a graduação, existem informações que o aluno precisa buscar fora da sala de aula, não só durante a disciplina, porque o conteúdo não é suficiente, segundo ela, para se obter uma boa formação.

Além do contato que teve com a cultura da LE em sala de aula, durante a graduação, P1 afirma ter contato com a língua por meio da Internet, jornais, revistas, filmes, músicas e livros de literatura. $\mathrm{Na}$ entrevista, P1 confirmou estas fontes de contato com a LE:

\section{Segmento 10}

"/... eu eu uso muito a internet /... é basicamente a internet que eu procuro quando eu (+) eu desconheço um tema e vou procurar tratar daquilo $(+)$ que é mais rápido (+) de de procurar do que livro $(+)$ mas livro também $(+)$ livro $(+)$ filme $(+)$ revista $(+)$ fita de vídeo" (Entr-P1)

Na opinião de PI, estes contatos são determinantes para o seu desempenho como professora de LE. Segundo ela, eles seriam uma alternativa para o professor que nunca foi ao exterior e que não tem um bom conhecimento da cultura da LE. Ela afirma, porém, que os filmes, por exemplo, apresentam muitos estereótipos da cultura do país e o professor deve ter um "filtro" para saber o que é real.

Ao ser questionada, durante a entrevista, a respeito de como o professor pode adquirir este "filtro", P1 afirma:

Segmento 11

"\%/ os livros esses materiais (+) mesmo filme traz uma visão (+) $\mathrm{um}$ estereótipo do que é a cultura (+) né" (+) e ai só uma boa formação do professor (+) uma conscientização maior que vai trazer isso /.../ ell acho que o professor ele tem que ter essa consciência pra (+) desmistificar o aluno (+) aqui a gente também tem (+) muita coisa boa $(+)$ por isso que eu falo essa (+) comparação entre as duas culturas (+) vai fazer eh: (+) 
criar um aluno crítico (+) com relação à outra /.../ muitas pessoas começam a aprender inglês às vezes muito cedo $(+)$ e: não tem (+) um objetivo ainda (+) ai desvaloriza a nossa cultura totalmente" (Entr-P1)

\section{Conclusão}

Passo agora a uma retomada da análise feita, ressaltando alguns aspectos considerados importantes no que se refere à prática do professor e suas crenças sobre o ensino da cultura enquanto vinculada à linguagem.

Um aspecto a ser ressaltado, é a percepção da dificuldade de P1 em obter material para o estabelecimento de uma comparação entre a cultura da LM e a cultura da LE. Na primeira categoria, pode-se observar que ela trabalha com a visão dos alunos ou com a visão do LD.

Percebe-se, neste ponto, que, apesar de a professora ter afirmado procurar ter contato com a cultura da LE por meio de Internet, jornais, revistas, filmes, músicas e livros de literatura, este contato pode não estar sendo suficiente para que ela tenha à sua disposição mais possibilidades de comparação entre as duas culturas em sala de aula.

Um outro aspecto observado é que P1 tem dificuldades quanto às dúvidas de vocabulário dos alunos. Nos momentos de incerteza quanto ao vocabulário, ele afirma que vai procurar posteriormente, porém, freqüentemente, isso não acontece. Este fato pode causar um baixo insumo cultural oferecido aos alunos, pois o desconhecimento de expressões e palavras pode limitar ou deter as explicações a respeito da língua e de como é o uso dessas expressões e palavras.

Pode-se verificar que, nas categorias, tanto no que se refere à prática da professora (como na questão a respeito das "favelas"). quanto ao uso da linguagem, há um problema na relação culturalinguagem. Por exemplo, a palavra "favela" está situada em um dado discurso comunitário, o da nossa língua materna, tanto que não é traduzível para o contexto da LE, e, para tal contexto, ela não serve. sendo outros termos mais apropriados para retratar a realidade da cultura estrangeira.

Aqui temos a linguagem expressando uma realidade cultural, que segundo Kramsch (1994), são idéias e fatos que expressam um 
conhecimento de mundo compartilhado. E, falando-se sobre a cultura como algo que ao mesmo tempo impõe e liberta, aqui vê-se a imposição de um conhecimento para que os aprendizes possam compreender a visão de mundo da cultura estrangeira.

Estes fatos são apenas exemplos que espelham o fato de que, para o estudo da relação cultura-linguagem é necessário ter a consciência de que, como afirma, Brown (1987), língua e cultura estão intrinsicamente ligadas e não se pode separar as duas sem perder o significado tanto de uma quanto da outra. Para a professora esta consciência é importante, porém não foi bem trabalhada na universidade, como ela mesma afirma.

Houve, portanto, uma falta de trabalho a respeito da consciência culturaí na formação pré-serviço, o que leva pode levar os professores formados a não privilegiarem este trabalho em sala de aula.

\section{Referências}

BROWN, D.H. Principles of Language Learning and Teaching. Englewood Cliffs, NJ: Prentice Hall Regent, 1987.

CONSOLO, D. A. The Ability for Communicative Language Use: an Overview. Revista de Letras, PUC-Campinas, Campinas, 18 (1/2) 8291, Dez 1999.

HINKEL, E. Culture in Second Language Teaching and Learning. Cambridge, UK: Cambridge University Press, 1999.

KRAMSCH, C. Context and Culture in Language Teaching. Oxford, UK: Oxford University Press, 1994.

KRAMSCH, C. Language and Culture. Oxford, UK: Oxford University Press, 1998. 

DE LIINGUA ESTRANGEIRA

LARSON, D. N.; SMALlEY, W. A. Becoming Bilingual: A Guide to Language Learning. New Cannan, CN: Practical Anthropology, 1972. ROSALDO, M. Towards an Anthropology of Self and Feeling. In R. Shweder \& R. Le Vine (orgs.) Culture Theory. Cambridge, UK: Cambridge University Press, 1984, p. 137-157.

THOMAS, J. Cross-Cultural Pragmatic Failure. Applied Linguistics, v. 4,1, p. $91-112,1983$.

THOMAS, J. Cross-Cultural Discourse as "Unequal Encounter": Toward a Pragmatic Analysis. Applied Linguistics, v. 5, 2, p. 226-235, 1984.

WATSON-GEGEO, K.A. Ethnography in ESL: defining the essentials. Reprinted from TESOL Quaterly, 22, p. 575-592, 1988. 